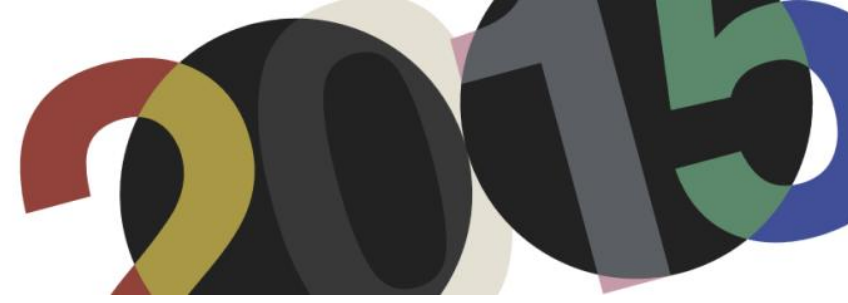

DOI: http://dx.doi.org/10.4995/LC2015.2015.523

\title{
La Villa Savoye. Permanencias y transformaciones
}

\author{
T. Carrau Carbonell
}

\author{
Escuela Técnica Superior de Arquitectura de Valencia
}

\begin{abstract}
Resumen: El Patrimonio del Movimiento Moderno y su conservación es un tema de actualidad, no solo desde el punto de vista teórico sino también desde el práctico, ya que la arquitectura de principios del s.XX lleva tiempo necesitando y asumiendo intervenciones para mantenerlas en pie. Se escoge como tema de estudio la vivienda, como arquetipo estudiado por los grandes del Movimiento Moderno y concretamente la villa Savoye como paradigma del Estilo Internacional. Así, con la investigación de este modelo se pretende sacar conclusiones para la conservación de la herencia de Le Corbusier asegurando su permanencia en las generaciones futuras. Se ha realizado un análisis de la villa Savoye por etapas de tiempo. Cada periodo se ha estudiado a través de unos parámetros comunes, obteniéndose una panorámica de la evolución de los elementos que la forman y de su globalidad. Los resultados de este análisis permiten hacer un estudio comparativo entre la villa original, la villa en sus distintas etapas y el estado actual, precisando qué es lo que realmente queda de la villa de 1930. De esta aportación surgen preguntas y reflexiones: ¿importa la conservación de la materia original en la permanencia de un hito? ¿es aceptable una máquina para habitar que no se puede habitar? ¿qué prevalece: la autenticidad arquitectónica o a la autenticidad histórica en la conservación de las obras de Le Corbusier?
\end{abstract}

Abstract: The Heritage of the Modern Movement and its conservation is a current topic, not only from the theoretical point of view but also from the practical, that is because the early twentieth century architecture has been needing and assuming interventions to keep up. It is chosen as a subject of study the housing, as well studied by the great archetype of the modern movement and specifically the Villa Savoye as a paradigm of the International Style. Thus, the investigation of this model is to draw conclusions for the preservation of the heritage of Le Corbusier ensuring its permanence in future generations. It has conducted an analysis of the Villa Savoye through stages of time. Each period has been studied through common parameters, giving an overview of the evolution of the elements that shape it and its entirety. The results of this analysis can make a comparative study between the original villa, the villa in its different stages and the current status, specifying what really remains of the villa of 1930. This contribution questions and thoughts arise: Does it matter the conservation of the original matter in the permanence of a milestone? Is it acceptable to inhabit a machine that you can not live in? Architectural authenticity prevails against the historical authenticity in the conservation of the works of Le Corbusier?

Palabras clave: Savoye; evolución; permanencia; intervención; patrimonio.

Keywords: Savoye; evolution; permanence; intervention; heritage.

\section{Una caja en el aire}

"La realidad en cuanto tal es desde sí misma constitutivamente abierta (...) Investigar lo que algo es en la

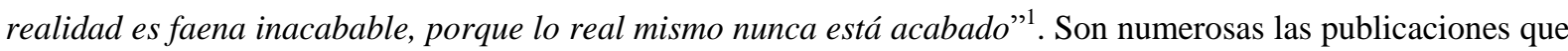
se han realizado sobre la Villa Savoye; desde el inicio de la historia de la villa en 1931 con un artículo publicado en Architecture Vivante ${ }^{2}$, hasta hoy con la recién editada "Les Heures Claires de la Villa Savoye"3 de Jean-

\footnotetext{
${ }^{1}$ Zubiri, Xavier: ¿Qué es investigar?. Discurso en la entrega del Premio Ramon y Cajal. Octubre 1982

${ }^{2}$ Le Corbusier \& Jeanneret. P: “Maison Savoye, Poissy”. En Architecture Vivante vol. 16. Paris: Jean Bodovici, 1931

${ }^{3}$ Savoye, Jean-Marc; Delhomme, Jean-Philippe: Les Heures Claires de la Villa Savoye. Paris: Éditions des Quatre Chemins, 2015
} 
Philippe Delhomme y Jean-Marc Savoye donde se recogen una serie de originales ilustraciones que reflejan la vida de los Savoye en la Villa. Entre tanto, se han sucedido investigaciones en profundidad como la de Josep Quetglas recopilada en "Les Heures Claires. Proyecto y arquitectura en la Villa Savoye de Le Corbusier y Pierre Jeanneret"4 o la de Jacques Sbriglio "Le Corbusier. La Villa Savoye" y muchos otros que han sido imprescindibles en la elaboración del presente trabajo. A pesar de que todos son estudios sobre el mismo objeto, cada uno de ellos arroja nuevas luces sobre la obra de Le Corbusier, temática que resulta una inagotable fuente de conocimiento dada la brillantez del personaje.

La investigación que a continuación se resume tiene como objetivo conocer lo que queda hoy de la villa original y Porqué, descifrando el Qué y el Cómo de las distintas intervenciones y sucesos en ella ocurridos desde su construcción hasta la actualidad. ¿Ha sobrevivido la Villa Savoye a los avatares del tiempo? ¿Sigue existiendo hoy la casa que Le Corbusier proyectó y construyó en Poissy hace más de ocho décadas?

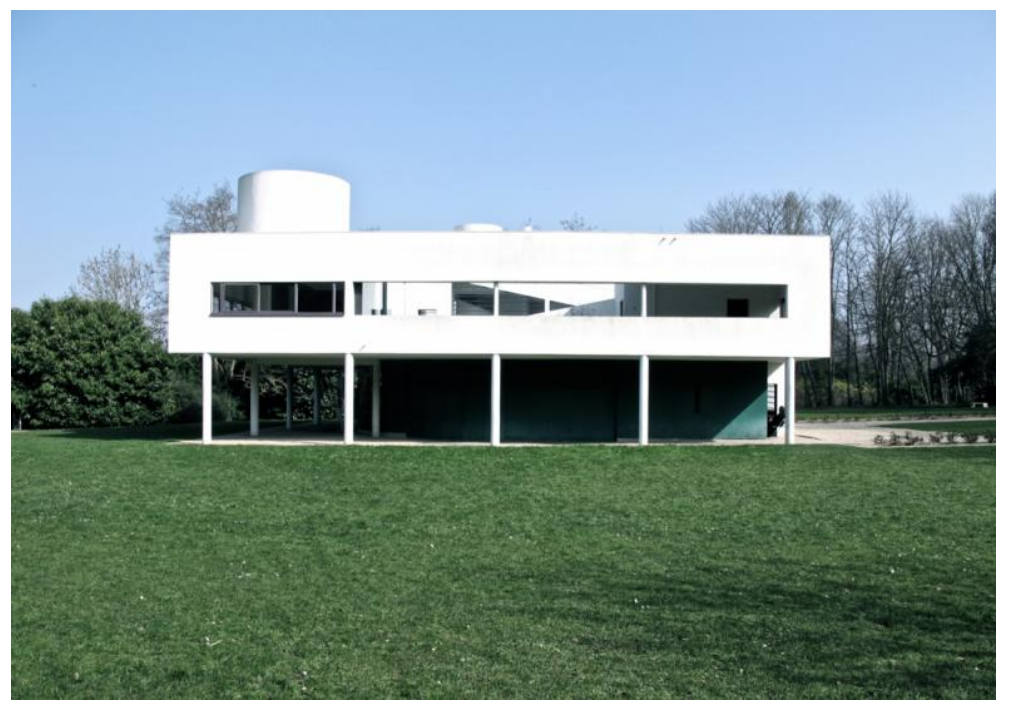

1. Imagen de la villa en 2014. Una caja en el aire.

\section{Evolución retrospectiva}

El análisis de la villa se ha realizado siguiendo una línea del tiempo; desde 1928 hasta 2014. La historia de la villa se ha estudiado por etapas. Cada etapa a través de siete parámetros: los personajes, el tiempo, el emplazamiento, la función, la envolvente, los espacios y recorridos y la materialidad. De este modo se obtiene una trayectoria en el tiempo de los elementos constituyentes de la villa. En cada uno de los periodos se ha recurrido a fuentes de la época que se analiza. Así, en la investigación de la villa de los años 30 ha sido de gran importancia el estudio de textos de Le Corbusier y en etapas posteriores la documentación de testigos de cada época. Una vez se ha pasado cada periodo por el tamiz de los parámetros, se ha hecho un análisis comparativo de los elementos que han pasado por la red, es decir, se han obtenido los elementos de la villa que han permanecido desde su construcción hasta hoy y los elementos que se han transformado, cuando y porqué.

\footnotetext{
${ }^{4}$ Quetglas, Josep: Les Heures Claires. Proyecto y arquitectura en la Villa Savoye de Le Corbusier y Pierre Jeanneret. Sant Cugat del Vallès : Associació d'Idees, Centre d'Investigacions Estètiques, 2008

${ }^{5}$ Sbriglio, Jacques: Le Corbusier. La Villa Savoye. Madrid: Abada editores, 2005
} 


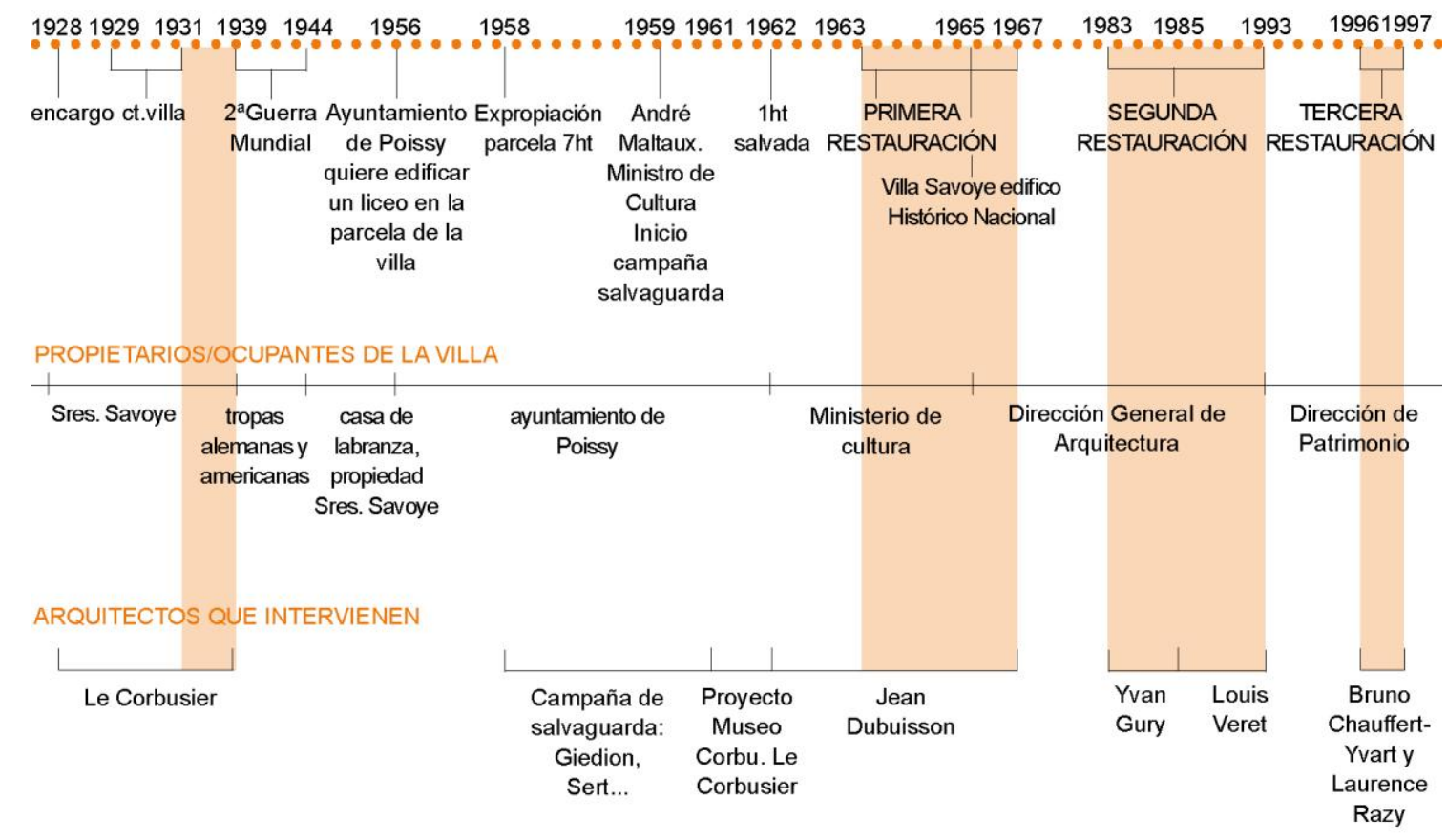

2. Línea del tiempo de la Villa Savoye.

\subsection{Una casa, una historia}

La historia de la villa comienza en 1928, cuando Pierre Savoye, director de una compañía de seguros, y su mujer Eugènie encargan a Le Corbusier una casa donde poder disfrutar los fines de semana lejos del ajetreo de la ciudad, en un amplio terreno a 30 km de París. A continuación, se señalan únicamente los aspectos o parámetros esenciales de cada periodo, necesarios para el entendimiento de su transformación en el tiempo y su estado actual.

\subsubsection{La Villa en 1931}

“La casa es una caja en el aire (...) debajo de la caja, pasando a través de los pilotes llega un camino de coches (...) del interior del vestíbulo arranca una rampa suave, que conduce casi sin darse cuenta, al primer piso, en donde transcurre la vida del habitante (...." ${ }^{\prime 6}$. Con la villa recién construida, Le Corbusier describe la casa recorriéndola, plasmando esa preferencia por la arquitectura árabe en cuanto a que se aprecia en movimiento. De esta descripción se obtienen dos claves de la villa original: la caja y el recorrido.

"Arriba en el lado norte de este cuadrado, se perciben cuerpos cilindricos, paredes con curvas libres...Estas partes superiores son de colores muy claros: azul, rosa y amarillo" "7. En este texto escrito por Julius Posener tras una visita a la villa en los años 30 , se encuentra otro aspecto esencial de la villa original; los colores de la envolvente.

\footnotetext{
${ }^{6}$ Le Corbusier: Precisiones respecto a un estado actual de la arquitectura y del urbanismo. Barcelona: Poseidón, 1978 p.p.158

${ }^{7}$ Posener, Julius: “La Maison Savoye à Poissy”. En Architecture d'Aujourd'hui No2, Paris:1930. p p. 20-21
} 


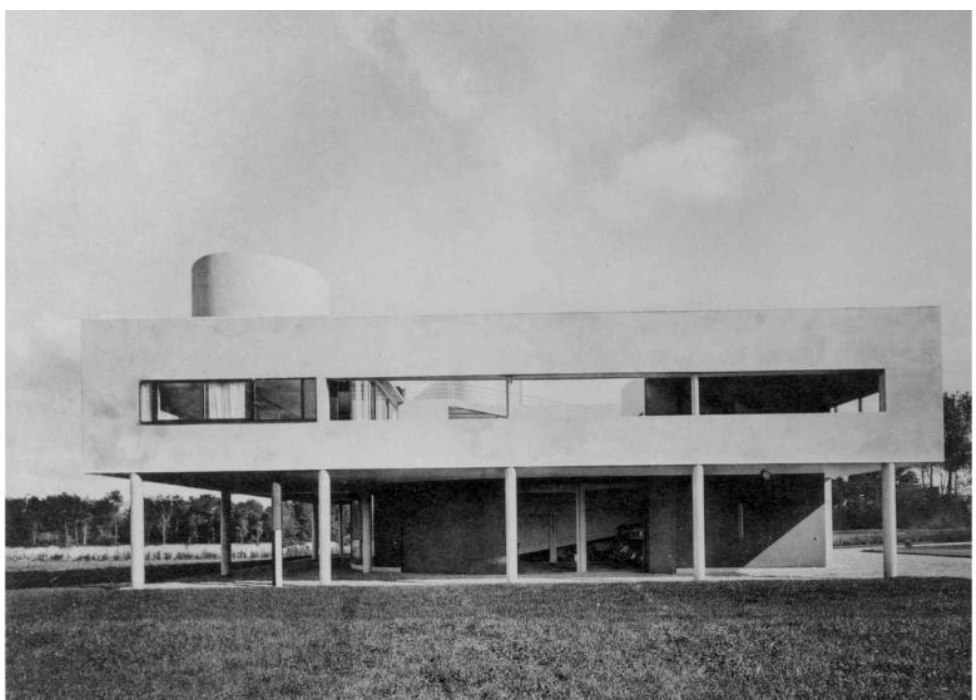

3. Imagen exterior de la villa en 1931. L2(17)4 @FLC-ADAGP

\subsubsection{Periodo de degradación 1938-1962}

Se trata de un periodo muy largo y cuyo comienzo está marcado por la Segunda Guerra Mundial, cuando los Sres. Savoye abandonan Paris. En este momento la villa pierde su FUNCIÓN de vivienda que ya nunca será recuperada. Durante la guerra fue ocupada por las tropas alemanas aprovechando su posición estratégica como punto de observación aéreo. Cuando finalizó la guerra la villa fue alquilada como casa de labranza para la explotación agrícola de los campos circundantes y en los últimos años de este periodo llegó a utilizarse como club de jóvenes antes de su clasificación como Monumento Histórico en el año 1962.

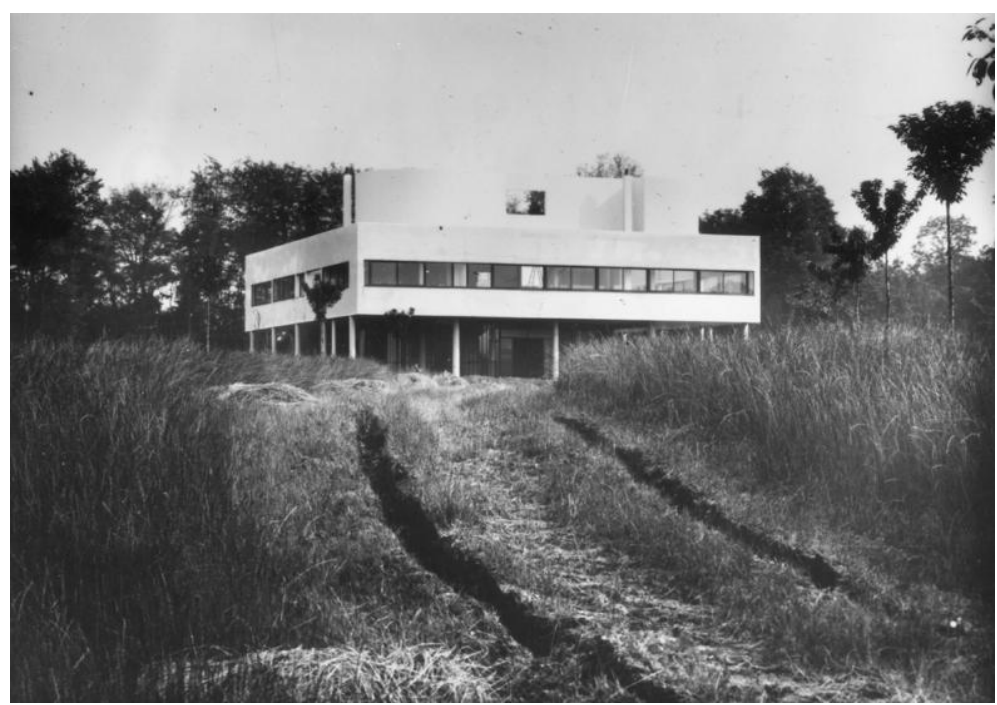

4. Villa en la década de los 50. L2(17)15. Fotógrafo: Marius Gravot. CFLC-ADAGP

A finales de los años 50, Poissy se convierte en una ciudad industrial motorizada por la industria del automóvil, con el consiguiente aumento demográfico. Esto significa que el vasto terreno que ocupa la parcela de la villa se convierte en un punto de mira para el Ayuntamiento de Poissy, que busca terreno donde construir los nuevos equipamientos. Tras un periodo de litigios entre los Savoye, el Ayuntamiento, Le Corbusier y grandes 
personalidades de la arquitectura, se expropian 6 hectáreas salvándose solo 1 hectárea en la que se encuentra la villa y la casa del jardinero. El resultado de este acontecimiento es la pérdida del entorno original de la villa, que a partir de este momento tendrá un liceo como vecino en lugar de amplias praderas y bosque.

"Tal vez, y tendría gracia la paradoja, los niños de la escuela cercana podrán pensar que se trata de la vivienda que han levantado, en su viaje a la Tierra, los habitantes de otro planeta, pues el optimismo progresista de Le Corbusier no se ha perdido, aunque la vivienda entera se haya convertido en una auténtica ruina"8. Respecto a la materialidad, este periodo significa el estado de máxima degradación de la villa siendo los acabados, "la carrocería" y las instalaciones, "el motor"10, las partes más afectadas, tal y como puede observarse en los esquemas de la imagen $n^{\circ} 5$.

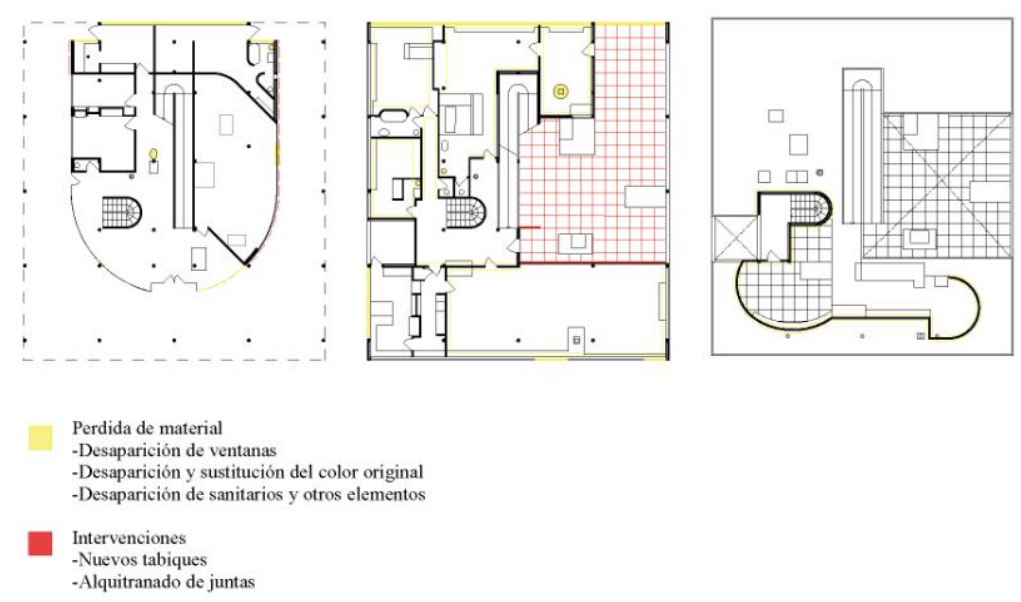

5. Estado de la Villa Savoye en la década de los sesenta, tras el periodo de degradación: enlucidos despegados del paramento, carpinterías e instalaciones rotas, elementos impropios, etc.

\subsubsection{Museo LC 1961-1965}

En los años 60 ante el deterioro de la villa, Le Corbusier insiste en la intervención inmediata para proteger a la villa de un empeoramiento material. Propone un nuevo uso como Museo Corbu, precisamente para protegerla y conservarla. Aunque este proyecto nunca se realizó por motivos burocráticos y económicos, es de gran interés porque supone lo que Le Corbusier hubiera hecho sobre su propia obra. Josep Quetglas dedica un capítulo en "Les Heures Claires" Corbusier. Quizás el mas relevante es la nueva puerta de acceso pivotante y esmaltada que hubiera supuesto un gran resalte en fachada, adecuado para el nuevo programa de museo. Además, asumiendo que iban a construir un liceo a pocos metros de la villa, propone que los visitantes accedan a pie y en sentido contrario al original para dejar así el liceo a la espalda y tener como fondo de perspectiva la villa. Propone una nueva paleta de colores

\footnotetext{
${ }^{8}$ Moneo, Rafael: “Una visita a Poissy”. En Arquitectura. No74. Madrid: COAM, 1965. pp. 35-41

${ }^{9}$ Le Corbusier: "El plano de la casa moderna". En Precisiones respecto a un estado de la arquitectura y del urbanismo. Barcelona: Poseidón, 1978. pp. 146

${ }^{10}$ ídem.

${ }^{11}$ Quetglas, Josep: Les Heures Claires. Proyecto y arquitectura en la Villa Savoye de Le Corbusier y Pierre Jeanneret. Sant Cugat del Valdès: Associaciò d'idees. Centre d'Investigacions Estètiques, 2008
} 
para el interior y la sustitución de las carpinterías por otras metálicas mas sencillas; "He preparado la habitación interior de la Villa Savoye en Museo Corbu (sin mobiliario). Mi contratista de albañilería no puede trabajar a destajo porque es bricolaje (es un hombre de confianza). La calefacción y la instalación eléctrica (que fue arrancada por completo) son elementos nuevos, igual que las ventanas (que simplificaré mucho)",12.

En definitiva, Le Corbusier introduce cambios en la villa que la actualizan y adaptan a las nuevas circunstancias, aunque conservando siempre la esencia del edificio. Le Corbusier muere en 1965 sin ver siquiera el comienzo de la primera restauración.

\subsubsection{Primera restauración 1963-1967}

El arquitecto encargado de la primera intervención en la villa es Jean Dubuisson, arquitecto jefe de Edificios Civiles y Palacios Nacionales en Francia. Aunque fue designado para la realización de este trabajo en 1963, el dossier con los trabajos pertinentes no se entregan hasta 1965, ejecutándose la obra entre 1965 y 1967.

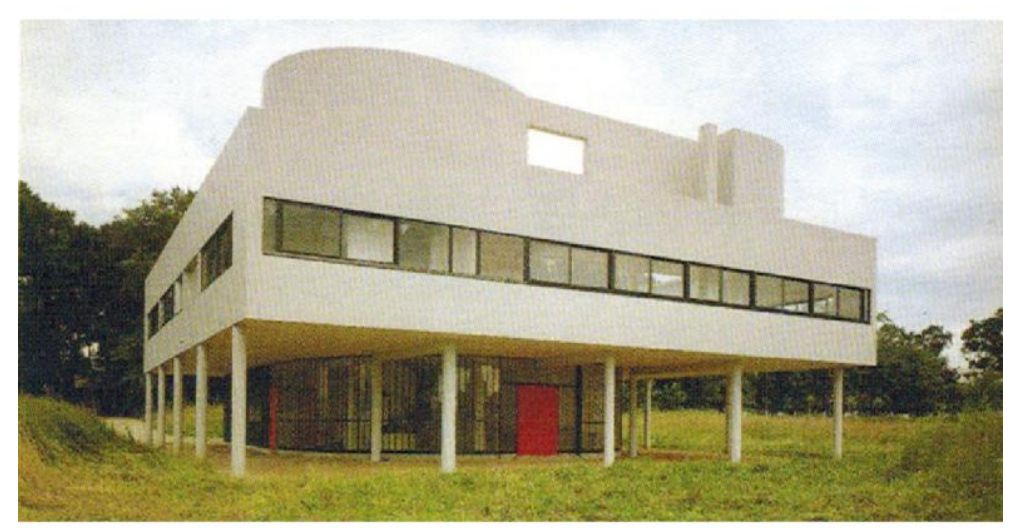

6. La villa Savoye tras la primera restauración

Tal y como se ve en la imagen $\mathrm{n}^{\circ} 6$, el resultado de la intervención es una villa con aspecto de nueva, en la que destacan dos elementos: el color blanco del solárium y la caja, y el color rojo de la puerta de acceso. Este último quizás represente la intención de hacer un guiño al proyecto de Museo Corbu, en que Le Corbusier resaltaba la puerta de acceso haciéndola pivotante y esmaltada.

\footnotetext{
${ }^{12}$ Carta de Le Corbusier a Claudius Petit, del 20 de junio de 1962. (FLC H1-12-24). En Quetglas, Josep: Le Corbusier y Pierre Jeanneret. Villa Savoye "Les Heures Claires" 1928-1962. Madrid: Ediorial Rueda S.L, 2004. Nota 30
} 

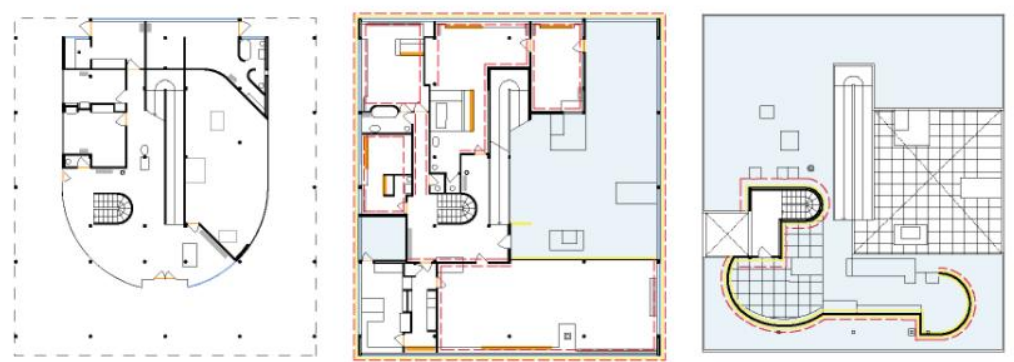

Demolición

-de tabiques

-del enlucido del solárium

Intervención

-pintura blanca en el exterio

-pinturas con tonos de la FLC en el interio

intervención en ventanas:

nuevas carpinterias metalicas

impermeabilización

reparación de puertas y armarios (madera)

cambio de instalaciones: calefacción y agua

7. Actuaciones durante la primera intervención global 1963-1967: impermeabilización de cubiertas, demolición de elementos impropios, sustitución por carpinterías metálicas, reparación de armarios y puertas, etc.

\subsubsection{Segunda restauración 1983-1993}

La falta de mantenimiento hizo necesaria una segunda intervención global. Algunos autores como T. Prudon afirman que ya desde 1977 se habían realizado algunos trabajos de mantenimiento, pero no es hasta 1983 cuando se plantea un trabajo de restauración global. En este caso, también el Arquitecto de Edificios Civiles y Palacios Nacionales del momento se encargó de la intervención.

Los objetivos eran dos: recuperar el esquema de color de la villa y resolver los problemas de impermeabilización. Para recuperar los colores se recurre a los tonos de la Fundación Le Corbusier sin reproducir los originales perdidos. En el comienzo de este periodo el solárium se pinta de tonos similares a los que describía Posener, aunque se acaba pintando de blanco. El mismo criterio se aplica a otros elementos desaparecidos como las luminarias; se escogen otras nuevas y distintas a las originales y se colocan en la misma posición que ocupaban en 1930. Las actuaciones abordadas en esta intervención se describen en la imagen n9.

En líneas generales, el resultado de esta actuación es la recuperación de la esencia de la villa; sus recorridos, transparencias y opacidades, sin hacer réplica de lo que falta. "Así, la Villa Savoye se ha convertido en un cuidado monumento histórico, inhabitado y por lo tanto no amueblado. Por eso, está bien protegido y accesible a los visitantes" ${ }^{\prime 3}$. Estas palabras de Alfred Roth publicadas en 1987, es una de las primeras impresiones de la villa recién abierta al público, en la que se intuye el olor a nuevo que debía tener la villa en ese momento. En 1993, la villa es asignada a la Dirección de Patrimonio y queda abierta al público en su totalidad.

\footnotetext{
${ }^{13}$ Roth, Alfred: “Bauherr war eine Baudame”. En Aktuelles Bauen Plan No9, 1987. pp. 30-33
} 

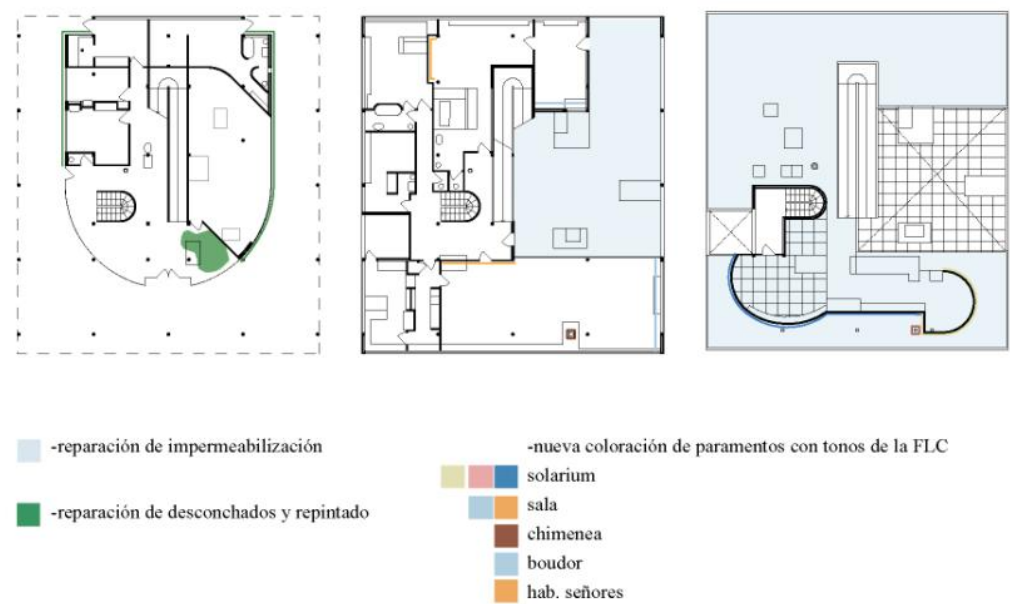

8. Actuaciones durante la segunda intervención global 1983-1993.

\subsubsection{Tercera restauración 1996-1997}

A finales de los 90 se propone una nueva intervención global con el objetivo de recuperar la atmósfera original de la villa y actualizarla en su uso como museo. Bruno Chauffert-Yvart y Laurence Razy son los encargados de esta actuación.

Para recuperar la atmósfera se recuperan los colores originales por medio de distintos estudios y catas con un criterio: solo se reproducen los colores de los que se tiene constancia, el resto se queda en blanco, tal y como describe Mourel Journel ${ }^{14}$ en la guía "Le Corbusier's Villa Savoye". Además, se reproducen los elementos originales perdidos en la etapa de degradación, como lámparas o interruptores, imitando los originales. Para adaptarla a su uso como museo, se sacrificaron algunas estancias quedando cerradas al público para uso de oficina o almacén, tal y como se grafía en los esquemas de planta de la imagen $n^{\circ} 10$. En los últimos años se han utilizado distintos recursos de merchandising que en algunos casos entorpecen la espacialidad de algunas salas.

\footnotetext{
${ }^{14}$ Morel-Journel, Guillemette; Ballot, Jean-Christophe: Le Corbusier's Villa Savoye. Paris: Editions du Patrimoine, 2000
} 

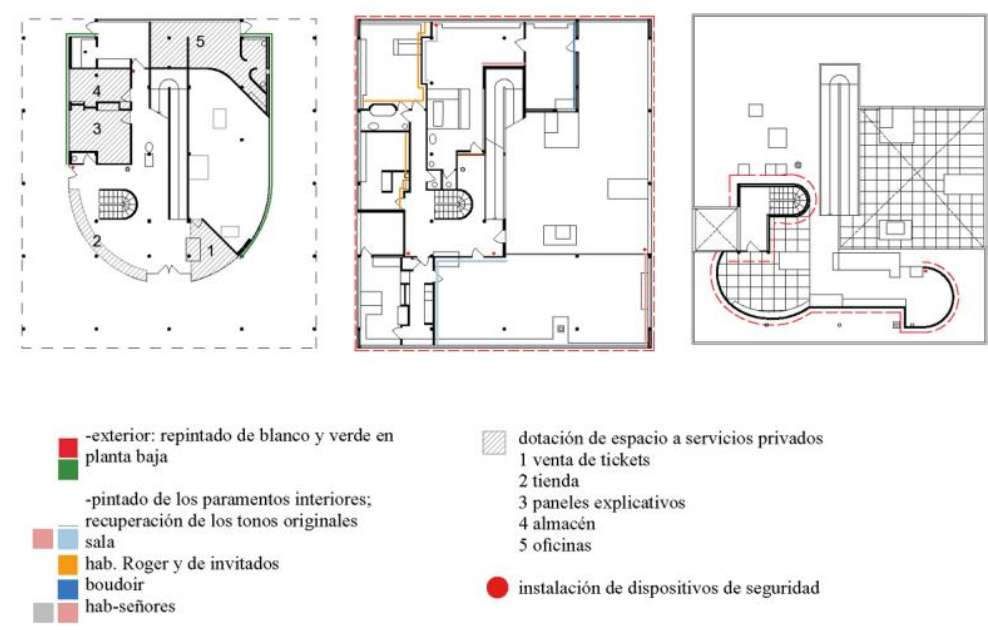

9. Actuaciones durante la tercera intervención global 1996-1997: recuperación de los colores originales y elementos desaparecidos.

\subsection{Paseo en el tiempo por la promenade architecturale}

Recogiendo todos los datos del análisis de la villa en sus distintos periodos: los personajes, el tiempo, el emplazamiento, la función, la envolvente, los espacios y recorridos y la materialidad, se hace un análisis comparativo de la villa entre las etapas clave, constatando e interpretando su evolución en el entorno, la envolvente y el interior. De esta comparativa se extraen los elementos que han permanecido y cuando se perdieron los que se transformaron.

\subsubsection{Situación en el entorno y el comienzo del paseo arquitectónico.}

"Otra cosa: la vista es muy bella, la hierba es una bella cosa y el bosque también: se les tocará lo menos posible. La casa se plantará en medio de la hierba como un objeto, sin dañar nada"15. Le Corbusier proyecta una casa para una parcela de 7 hectáreas de extensión, rodeada de bosque. La ubicación en medio de la parcela está cuidada y estudiada. Le Corbusier insistió mucho en la preservación del espacio abierto alrededor de la villa "si el parque es retirado, todo está perdido" 16 .

El análisis de una foto aérea de 1930 y otra de la actualidad muestra los cambios significativos que han sucedido en entorno, como consecuencia principal del aumento demográfico de Poissy. En el origen, la villa y la casa del jardinero estaban solas, sin edificaciónes alrededor, una cerca limitaba las 7 hectáreas de parcela. Ahora una valla y unos metros de cerca en el lado sureste limitan la propiedad de 1 hectárea. Así mismo cambia la extensión de prado y bosque y con ello las vistas desde la villa. En el origen, estaba colocada dominando todo el terreno en todo su perímetro y hasta un horizonte lejano, libre de obstáculos. Actualmente ese radio se ha reducido de forma considerable, por lo que las vistas tienen un horizonte más cercano que puntualmente deja pasar el cordón de árboles llegando a ver el edificio vecino, como se plasma en la imagen ${ }^{\circ} 10$.

\footnotetext{
${ }^{15}$ Le Corbusier; Boesiger, Willy: Le Corbusier et Pierre Jeanneret. Oeuvre complète de 1929-1934. Zurich : Les Editions d'Architecture, 1973. P.p.24

${ }^{16}$ Le Corbusier: FLC H1-12-229 en Murphy, D. Kevin: "The Villa Savoye and the Modernist Historic Monument” . En Journal of the Society of Architectural Historians. Edición digital: University of California Press, 2002. P.p.76
} 
¿Qué hacer cuando el tiempo ha modificado de forma irreversible el entorno de una obra? ¿se pierde en cierto modo su originalidad? Este tipo de cambios, exigen la aceptación de los mismos y su uso como herramienta de evolución, igual que Le Corbusier hizo en su proyecto de Museo Corbu. En el conocimiento y la imaginación del arquitecto está la clave para hacer posible la preservación un obra del pasado, asegurando su permanencia en el fututo y acogiendo la realidad presente. "No hay permanencia sin transformación"17.
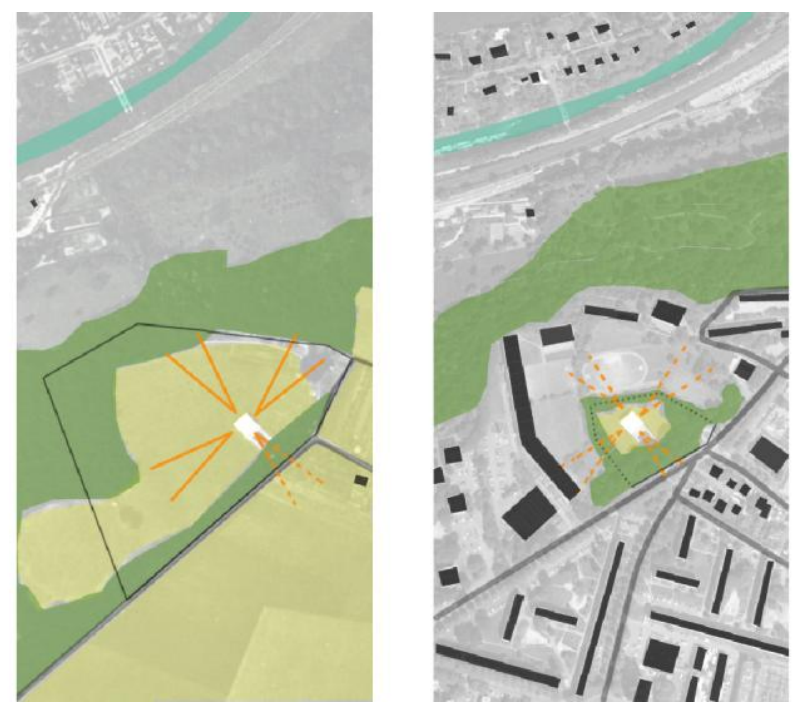

10. Esquema comparativo del entorno 1930 y 2014: se señala la extensión de prado, bosque y caminos, edificación y límites, vistas.

En el análisis del entorno próximo, donde comienza la promenade architecturale, se observa que con el paso del tiempo, o más bien de las intervenciones, la villa se aproxima cada vez más a la imagen original; una evolución retrospectiva. Aunque el contexto quedó definitivamente modificado con la expropiación del terreno, con la segunda intervención se consigue recuperar en cierto modo la sensación original de estar en un gran prado limitado por un bosque, mediante mecanismos de replantación de árboles a modo pantalla. De este modo, en cota 0 parece que la villa está implantada en un frondoso jardín sin rastro de edificios vecinos. Por otra parte, el original acceso para los coches en forma de horquilla, se perdió como tal al perderse la función de vivienda. En la última intervención este camino de grava se ha recuperado, replantando incluso el tipo de jardinería que le acompañaba en el origen, y aunque su uso ya no es rodado, el visitante actual puede hacer el recorrido original a pie.

\footnotetext{
${ }^{17}$ Bosch, Ignacio: extracto de la conferencia "La ruina como valor añadido". Valencia: 2014
} 

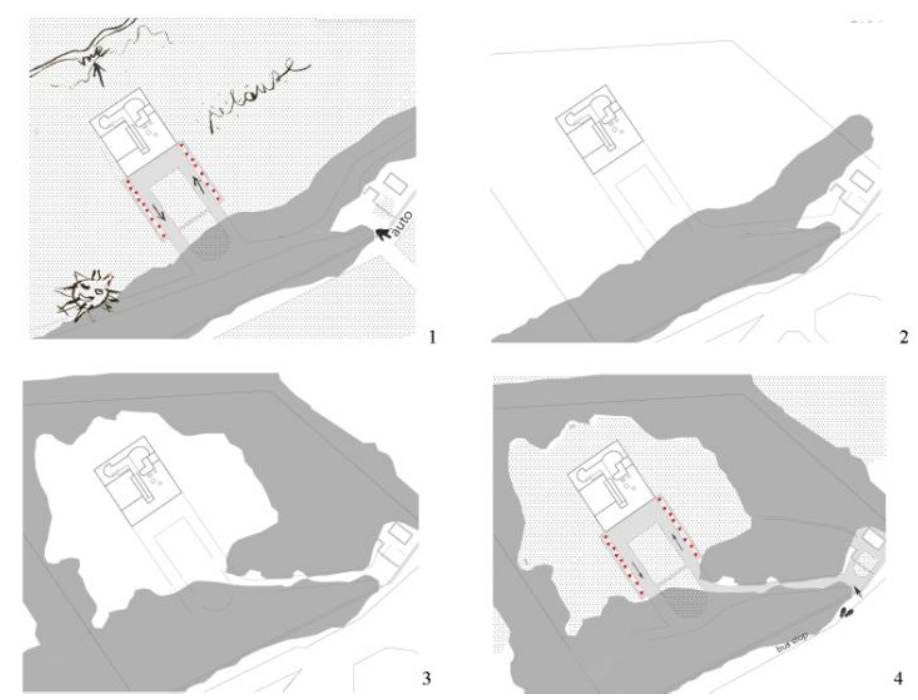

11. Esquema evolución del entorno próximo; 1: año 1930, 2: primera intervención, 3: segunda intervención, 4: tercera intervención: recuperación de la imagen idílica de la villa de 1930

Tras una visita a la villa Álvaro Siza escribió "Mucho del encanto de la Villa Savoye -de la Arquitectura- tiene su origen en una interrumpida y precaria complicidad entre los que la realizan: promotores, constructores, proyecto. Su continua degradación refleja la imposibilidad de mantener ese equilibrio encantador, sin dejar de buscarlo. No sabemos qué dioses la habitan. Como un templo Japonés, se recompone antes de resquebrajarse" 18

Quizás sea la captura del tiempo, lo que verdaderamente se busca en esta villa, como ocurre en otras intervenciones en obras del Movimiento Moderno ¿Cuál es el sentido de la búsqueda del origen y congelación en el tiempo, si el tiempo pasa irremediablemente?

\subsubsection{La envolvente}

El estudio de la envolvente conduce a la reflexionar sobre la evolución no retrospectiva de las fachadas, especialmente en lo que se refiere al solárium.

Le Corbusier pudo concebir el solárium como comienzo o fusión con el aire, las nubes y el cielo. El arquitecto describe la villa como una caja blanca flotando; por abajo flota sobre una sombra y por arriba el solárium desaparecería mediante el color; mediante tonos que podrían fundirse con los de un atardecer. Esta hipótesis estaría respaldada por los dibujos originales de las fachadas en los que Le Corbusier solo pinta de blanco la caja, por la descripción anteriormente comentada de Posener y por la maqueta original que guarda el MOMA, en la que el solárium es de tonos rojizos, azul y amarillo. Si bien, respecto a este último criterio, se han escrito hipótesis ${ }^{19}$ que apostarían por una villa blanca en su exterior.

\footnotetext{
${ }^{18}$ Siza, Álvaro: Textos. Madrid: Abada ediciones, 2014

${ }^{19}$ Quetglas, Josep: “Algo sobre el color de la arquitectura de Le Corbusier”. En Arquitectura №358. Madrid: COAM, 2009. pp.93-97: Josep Quetglas escribe entorno a algunas villas de la época purista de Le Corbusier: "Fuera, el edificio es inexpresivo, discreto, reservado, monocromo...En el interior, policromía imprevisible, sorprendente, impactante" Sobre la maqueta que Le Corbusier hace para la Exposición del MoMA de Nueva York con colores en el exterior "una maqueta es un objeto, no una arquitectura, no produce desplazamientos del observador"
} 
La dicotomía aparece revisando la historia de la villa, cuando en los años 60 se pica todo el revestimiento del solárium perdiéndose cualquier prueba del color que pudiera existir. Por otro lado, la posible persecución de una villa ideal, atendiendo el criterio de Viollet-Le-Duc de la unidad de estilo y restitución del objeto a un estado que quizás no haya existido nunca, puede llevar a pensar en un solárium blanco. Se trata de la casa que culmina la época purista de Le Corbusier, es el último eslabón de una serie de viviendas de fachada blanca.
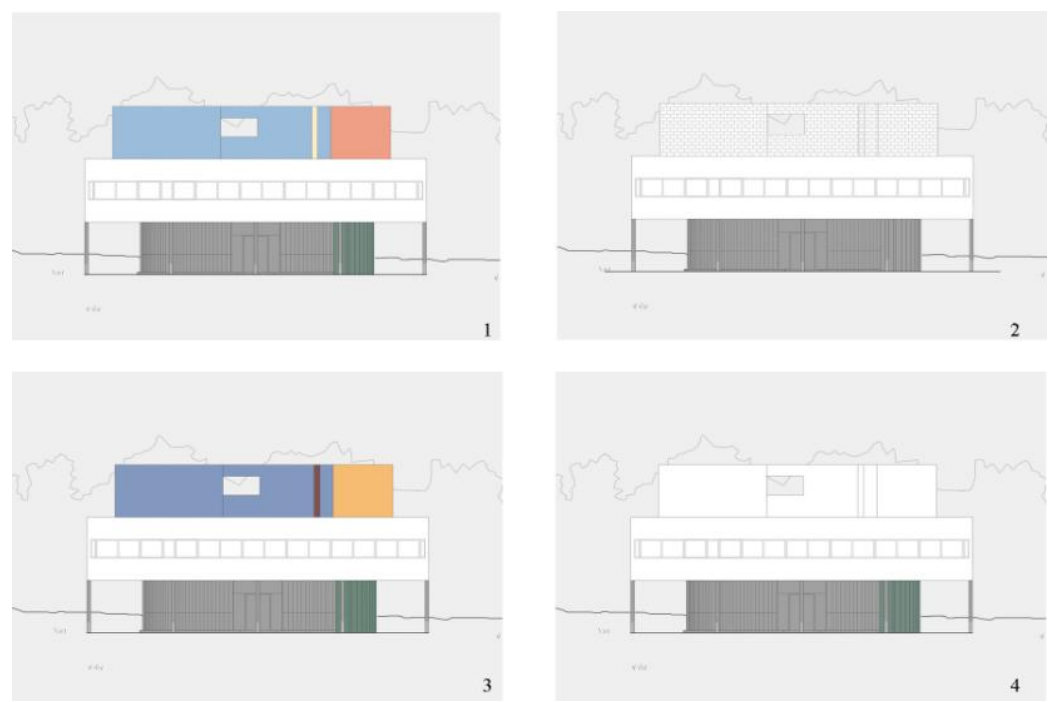

12. Esquemas evolución fachada noroeste; 1. año 1930, colores según maqueta original, 2. La villa durante la primera intervención sin enlucido en el solárium, 3. La villa tras la intervención de Ivan Gury a finales de los 70, 4 la villa tras la tercera intervención global.

\subsubsection{El interior}

Desde el punto de vista material, el interior de la villa ha evolucionado de forma similar al entorno; poco a poco se han borrado las huellas del tiempo. Durante la segunda intervención de principios de los 90 se actuó en la villa sin recurrir a la restauración en estilo; se utilizaron nuevos elementos para sustituir lo que se había perdido. Por ejemplo, en la sala, las paredes inicialmente rosa se pintan de color azul, la chimenea originalmente blanca se pinta de color terre hombre, se sustituyen las luminarias downlight de la entrada por uplight, etc. Esto levantó algunas polémicas entre los arquitectos conservacionistas como Lion $\mathrm{Krier}^{20}$, que vieron esta actuación como un ataque a la originalidad de la obra. Sin embargo, en la última intervención global hay un cambio de criterio; las paredes se han repintado con los tonos originales, las lámparas perdidas se han reproducido, etc. Se recurre, otra vez, a la restauración en estilo iniciada en el s.XIX con Violet-le-Duc, devolviendo la villa a lo que debió de ser. ¿Qué hubiera hecho Le Corbusier? La respuesta podría obtenerse en el Pabellón Suizo. El esquema de color original de los años 30 cambia por completo en la modificación que Le Corbusier hace en los años 50. Este y otros estudios de color están publicados en "The Architectonic Colour. Polychromy in the Purist Architecture of Le Corbusier" de Jan de Heer ${ }^{21}$. En ellos se observa que el arquitecto suizo actualizaba sus propias obras sin retenerlas o paralizarlas en un tiempo pasado. Le Corbusier valoraba su propia obra, como queda reflejado en sus escritos en la movilización mundial que organizó para el salvamento de la villa Savoye. Esto no es incompatible

\footnotetext{
${ }^{20}$ Krier, Lion: "SOS. Villa Savoye". En Architectural Design N5/6. London: 1992

${ }^{21}$ De Heer, Jan: The Architectonic Colour. Polychromy in the Purist Architecture of Le Corbusier. Rotterdam: 010 Publishers, 2009.
} 
con la evolución o actualización de su obra, como demostró en el Pabellón Suizo prescindiendo de la "Restauración en Estilo".
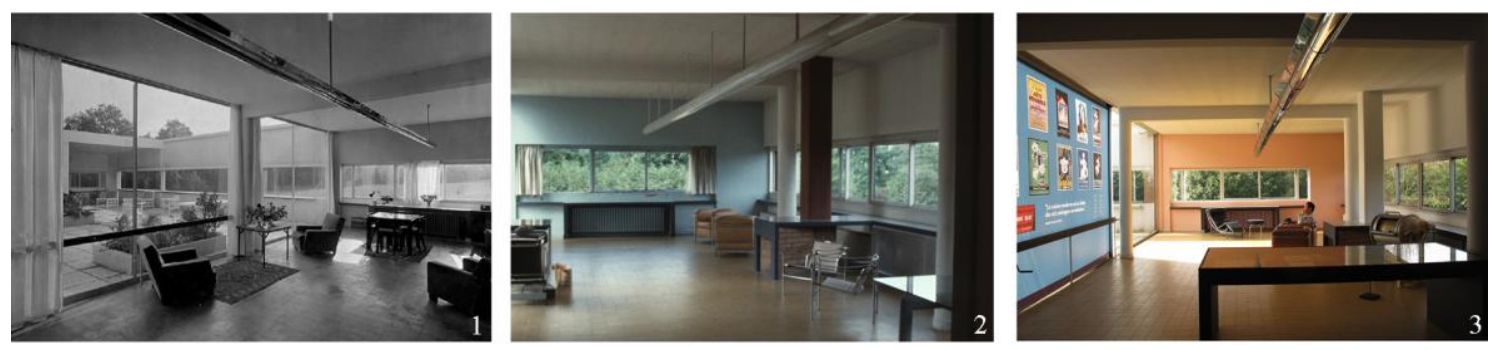

13. Evolución de la sala: 1 villa 1930. FLC L2(17)98. Fotógrafo: Marius Gravot. CFLC-ADAGP., 2. la sala en 1990, 3. La sala en 2014

\section{Villa Savoye ¿Indestructible?}

En una visita a Poissy hace menos de un año, me pude encontrar directamente con la Villa Savoye y preguntarme quien era. Tras recorrer el corto pero frondoso camino, entre árboles y vegetación y dejando atrás la casa del jardinero, la villa se aparece ante el visitante. La sensación es la de hallar una caja blanca atemporal. ¿Desde cuando estas aquí? El tiempo se detiene. Es un volumen tan limpio, que da igual que el enlucido esté sucio. Es limpia. Destila pureza, rotundidad.

Una vez dentro ya, todavía entre verdes y brillos, te encuentras con la rampa, empiezas a descubrir la arquitectura, comienzas la promenade architecturale, una arquitectura que se abarca paso a paso. Ya lo contó Giedion; "es imposible abarcar la Villa Savoye a primera vista, desde un solo punto de observación: es literalmente una construcción según el principio espacio-tiempo" ${ }^{22}$. En palabras de Sáez de Oiza, "la arquitectura es una secuencia de relaciones espaciales percibidas en función de las experiencias vividas y promesas de espacios por ver" ${ }^{23}$. Estas palabras se entienden bien hoy en la Villa Savoye, donde el espacio se construye con el tiempo, donde la arquitectura se entiende en movimiento tal y como prefería Le Corbusier.

“La arquitectura es el juego sabio, correcto y magnífico de los volúmenes bajo la luz” 24 . En la villa Savoye entendí, aprehendí la conocida frase de Le Corbusier. Allí estaba la luz correteando por las habitaciones, dibujando alfombras en el suelo, cambiando en cada instante, presentando a el Tiempo como su más fiel amigo. La emoción de la arquitectura que Le Corbusier pensó y proyectó para esta casa, están allí.

\footnotetext{
${ }^{22}$ Giedion, Siegfried: Espacio, Tiempo y Arquitectura. $5^{\text {a }}$ ed. Barcelona: Científico-Médica S.A, 1979

${ }^{23}$ Sáenz de Oiza, Francisco Javier: Tres arquitecturas. Entrevista TVE. Noviembre, 1990

${ }^{24}$ Le Corbusier: Vers une archicteture. Buenos Aires: Poseidón, 1978. pp.16-17
} 


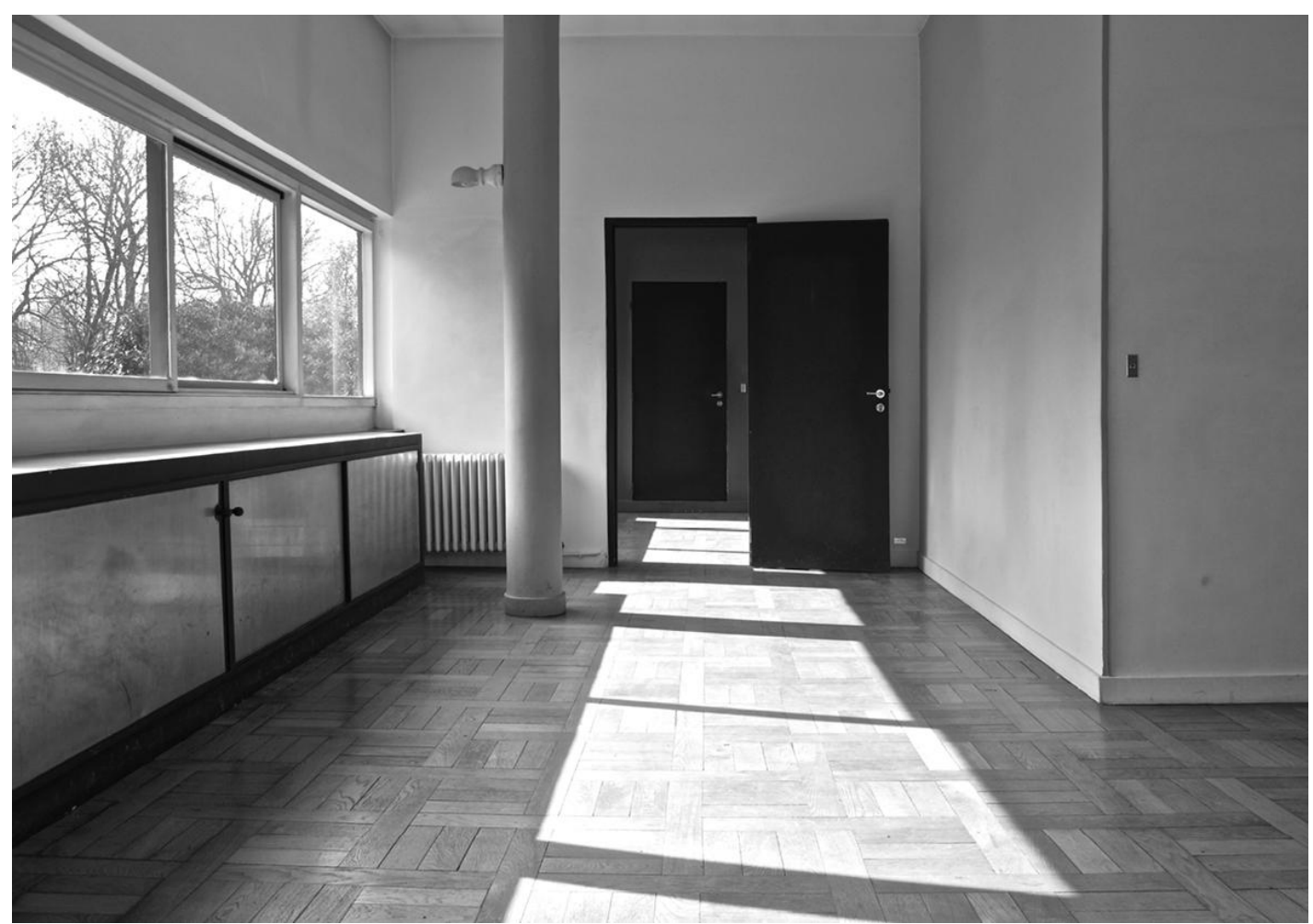

14. La Villa Savoye año 2014; juego de volúmenes bajo la luz.

Cada día el sol es atrapado por las rendijas de la villa Savoye. Se trata de una caja horadada en su cintura, por lo que recibe luz de norte, de sur, de este y de oeste. La luz de norte ilumina el espacio con luz difusa, y la de sur dibuja las horas en las estancias. Además, en la villa hay luz vertical, luz que entra desde el cielo solo a determinados espacios, como linternas apoyadas en el techo que hacen que te detengas y mires y busques. " $L a$ LUZ SÓLIDA en visible movimiento, danzando sobre una invisible LUZ DIFUSA en reposada quietud" ${ }^{25}$. La luz entra en vibración con el material que alumbra. En el interior, la luz juega con el blanco, lo vuelve gris, amarillo, lo vuelve de todos los colores. Algo así como el blanco de Sorolla, blanco que se pinta con gris, con azul, con rosa. Esta blancura coloreada la encontramos en la villa: "La blancura del blanco casi nunca es blanca: es casi siempre transformada por la luz y por lo que está cambiando: el cielo, las nubes, el sol y la luna" ${ }^{26}$.

\section{Conclusiones. La permanencia de un hito}

Teniendo como panorámica la evolución de la villa desde que se construyó hasta hoy, surgen una serie de reflexiones entorno al modo en que se ha conservado. La villa se ha mantenido más como imagen y concepto, que como testimonio material de una época ¿Es posible el mantenimiento de la herencia de Le Corbusier como testigo material fiel de construcción de principios del siglo XX, como parte de la historia? Según Wessel de Jonge, esto no es solo posible sino también necesario; "El Movimiento Moderno ha sido una inflexión fundamental en la historia de la edificación. Según mi punto de vista, fundiendo los conceptos sociales y

\footnotetext{
${ }^{25}$ Campo Baeza, Alberto: La idea construida. Buenos Aires: Editorial Nobuko, 2009. p.p 30

${ }^{26}$ Meier, Richard: publicación digital en www.richardmeier.com "White is the most wonderful color because within it you can see all the colors of the rainbow. The whiteness of White is never just White: it is almost always transformed by light and that which is changing; the sky, the clouds, the sun and the moon"
} 
tecnológicos de una época el Movimiento Moderno iguala la originalidad fundamental de la arquitectura romana y, según las publicaciones de Viollet-le-Duc, de la gótica. El segundo punto es que la arquitectura contemporánea perdería una parte esencial de su historia si no salvaguardamos algunos de estos edificios" 27. Andrés Martínez Medina también apoya la preservación de las huellas del tiempo en este tipo de arquitectura; "Borrar las huellas del tiempo de la arquitectura moderna impide que se las sitúe adecuadamente en el devenir del tiempo y de la historia. Acusar el tiempo no es volverse ruina. El tiempo debería reflejarse en las obras o las obras dejarán de ser de nuestro tiempo, habitarán una dimensión virtual"28. Sin embargo, teniendo como ejemplo la trayectoria de este Monumento, surge una pregunta; ¿importa el valor del Tiempo en una obra del Movimiento Moderno? ¿permiten los edificios del M.M mantener la pátina del tiempo como lo permiten las sólidas construcciones romanas? Se encuentra una posible respuesta a estas reflexiones en el entendimiento de la Villa Savoye como “machine à habiter”. Le Corbusier entendió en la máquina una nueva forma de proceder, " $E l$ paquebote es la primera etapa en la realización de un mundo organizado de acuerdo con el espíritu nuevo"29. Un máquina, para que funcione está en continuo cambio, una máquina no funciona siempre con las mismas piezas. La Savoye, como cualquier máquina requiere una constante renovación y mantenimiento de sus piezas para que funcione. Si por el contrario, se pretende conservar una máquina como reliquia, las piezas se arreglan y se reparan dejando el olor a viejo aunque esté implícita la pérdida de su función.
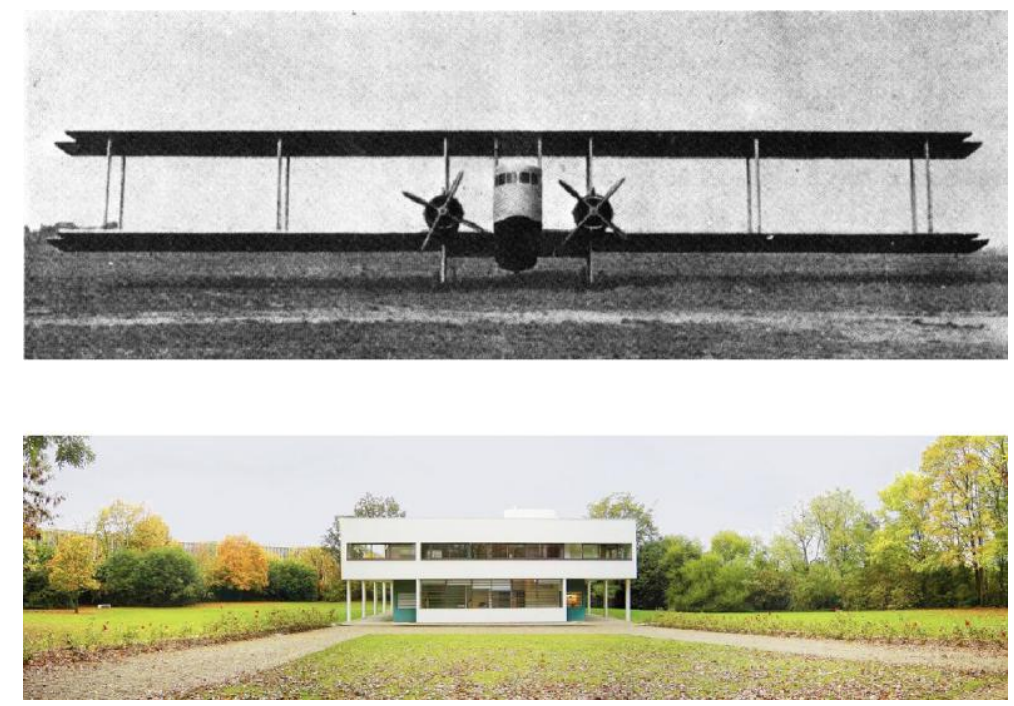

15. Le Corbusier: la máquina de volar y la máquina de habitar

Hoy pocos materiales originales quedan. A los problemas propios del abandono se suman las patologías originales de la obra. Le Corbusier investigó y experimentó en su obra. Esto explica la existencia de algunas "irregularidades" como goteras y otros problemas de humedad que tardaron en resolverse, haciéndose precisa la sustitución de algunos materiales originales. Además, las soluciones perecederas propias de la industrialización duran una serie de años, no una eternidad. A pesar de esta realidad, la Villa Savoye permanece hoy como modelo

\footnotetext{
${ }^{27}$ De Jonge, Wessel. En Ribera, David: Dios está en los detalles .Valencia: General de ediciones de arquitectura, 2012. p. $74-$ 75

${ }^{28}$ Martínez Medina, Andrés: "Las Huellas del tiempo en la arquitectura moderna intervenida”. En Conferencia Internacional CAH20thC. Criterios de Intervención en el Patrimonio Arquitectónico del Siglo XX. Madrid: Secretaría General Técnica, Ministerio de Cultura, 2011
} 
de una Nueva Arquitectura, como hito del Estilo Internacional. En la búsqueda del porqué, llego siempre a los mismos conceptos: "la Promenade", la Luz, el Tiempo. La villa Savoye se entiende tan bien hoy como ayer. "Los universales trascienden, crean un espacio regido solo por la geometría y la proporción, mas allá de todo lugar particular. Y trazan un tiempo eterno (...) Luz, Gravedad y Proporción significan Tiempo, Orden y Belleza. Se entiende, se experimenta que el valor no está en los materiales, en lo material, sino en los intangibles, en la idea, en lo espiritual" 30 escribe Alberto Burgos.

Esta reflexión bien puede plasmarse a través de una colección de lienzos pintados por Le Corbusier en los años veinte. Son una serie de cuadros, inspirados en aquella colección de Monet, en los que representa las mismas formas con distintas tonalidades y combinaciones de color. Se comprende bien la permanencia de la villa en el tiempo: son distintos los tonos de la paleta para cada cuadro, pero siempre dibujan las mismas formas. En la villa, son distintas las intervenciones, los acontecimientos que le hace pasar el tiempo, pero es siempre la misma Idea, es siempre la misma Villa. Quizás sea esto lo que de verdad importa, más el Qué y menos el Cómo: que las palabras de Le Corbusier sigan vivas en sus edificios, que sus obras sigan siendo una fiel representación de sus ideas: "Se utiliza la piedra, la madera, el cemento, y con esos materiales se levantan casas, palacios: eso es construcción, el ingenio trabaja. Pero, de pronto me conmovéis, me hacéis bien, soy dichoso y digo: es bello. Esto es arquitectura. El arte está aquí" ${ }^{31}$.
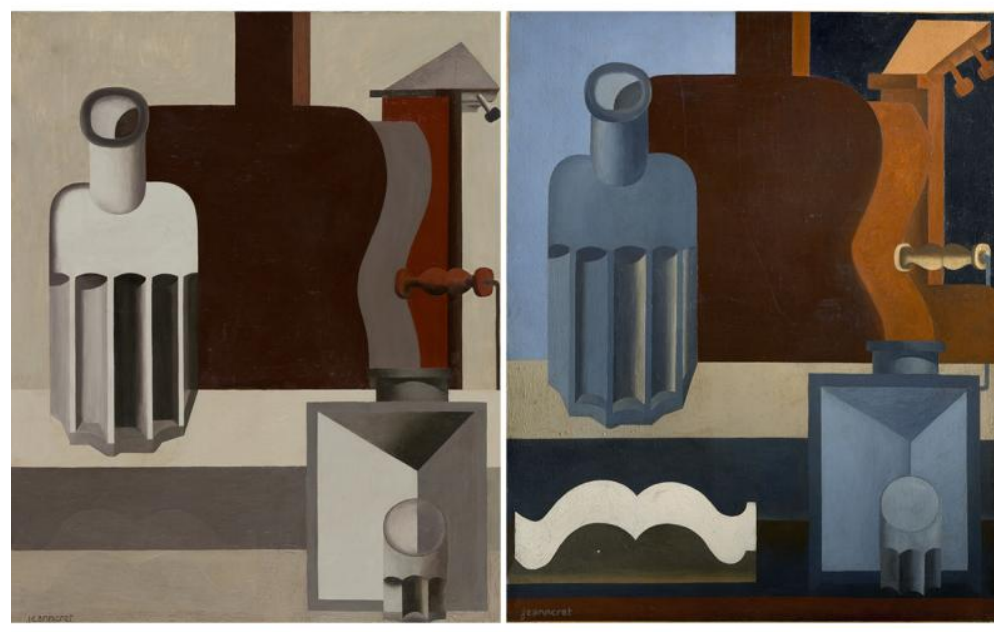

16. Guitarra vertical, 1920. Le Corbusier. Peinture FLC 138 @FLC-ADAGP. Peinture FLC 174 @FLC-ADAGP

\section{Agradecimientos}

A mis directores de tesis, así como a la Escuela Técnica Superior de Arquitectura de Valencia. A mi familia y amigos.

\section{Procedencia de imágenes}

$1,2,5,7,8,9,10,11,12,13.3,14$; realizadas por la autora del presente artículo.

${ }^{30}$ Burgos, Alberto: “Top. (desde 1992) Esencialidad. More with less". En Campo Baeza, Alberto: Alberto Campo Baeza. Arquitectura 2001-2014. Valencia: Ed. General Ediciones de Arquitectura, 2014. p.p 11

${ }^{31}$ Le Corbusier: Vers une Architecture. Buenos Aires: Editorial Poseidón, 1978. pp.123 
3; L2(17)4 @FLC-ADAGP. La Fondation Le Corbusier

4; L2(17)15. Fotógrafo: Marius Gravot. OFLC-ADAGP. La Fondation Le Corbusier

6; Torres Cueco, Jorge; Le Corbusier mise au point. General de ediciones de arquitectura. Memorias Culturales. Valencia, 2012

13.1; FLC L2(17)98. Fotógrafo: Marius Gravot. OFLC-ADAGP. La Fondation Le Corbusier

13.2; Torres Cueco, Jorge: 1990

15; OFLC-ADAGP. Le Corbusier: Vers une Architecture. Buenos Aires: Editorial Poseidón, 1978, p.p 59 / Burgos, Alberto: fotografía panorámica de la Villa Savoye.

16; Peinture FLC 138 @FLC-ADAGP. Peinture FLC 174 @FLC-ADAGP. La Fondation Le Corbusier

\section{Bibliografía}

Boesiger, Willy; Le corbusier: Le Corbusier et Pierre Jeanneret. Oeuvre complète de 1929-1934. Zurich: Les Editions d'Architecture, 1973

Burgos, Alberto: “Top. (desde 1992) Esencialidad. More with less". En Campo Baeza, Alberto: Alberto Campo Baeza, Alberto; Arquitectura 2001-2014. Valencia: Ed. General Ediciones de Arquitectura, 2014

Campo Baeza, Alberto: La idea construida. Buenos Aires: Editorial Nobuko, 2009.

Curtis, William J.R; Ideas and forms. London: Phaidon, 1986

De Heer, Jan: The Architectonic Colour. Polychromy in the Purist Architecture of Le Corbusier. Rotterdam: 010 Publishers, 2009.

De Jonge, Wessel. En Ribera, David: Dios está en los detalles .Valencia: General de ediciones de arquitectura, 2012.

Frampton, Kenneth: Le Corbusier. Barcelona: Editorial Akal, 2001.

Giedion, Siegfried: Espacio, Tiempo y Arquitectura. $5^{\text {a }}$ ed. Barcelona: Científico-Médica S.A, 1979

Krier, Lion: "SOS. Villa Savoye". En Architectural Design No5/6. London: 1992

Le Corbusier: Precisiones respecto a un estado de la arquitectura y del urbanismo. Barcelona: Poseidón, 1978

Le Corbusier \& Jeanneret. P: "Maison Savoye, Poissy". En Architecture Vivante vol. 16. Paris: Jean Bodovici, 1931

Le Corbusier: Vers une Architecture. Buenos Aires: Editorial Poseidón, 1978

Martínez Medina, Andrés: "Las Huellas del tiempo en la arquitectura moderna intervenida". En Conferencia Internacional CAH2OthC. Criterios de Intervención en el Patrimonio Arquitectónico del Siglo XX. Madrid: Secretaría General Técnica, Ministerio de Cultura, 2011

Moneo, Rafael: "Una visita a Poissy”. En Arquitectura. N74. Madrid: COAM, 1965.

Morel-Journel, Guillemette; BALLOT, Jean-Christophe: Le Corbusier's Villa Savoye. Paris: Editions du Patrimoine, 2000

Murphy, D. Kevin: "The Villa Savoye and the Modernist Historic Monument" . En Journal of the Society of Architectural Historians. Edición digital: University of California Press, 2002

Quetglas, Josep: "Algo sobre el color de la arquitectura de Le Corbusier". En Arquitectura No358. Madrid: COAM, 2009.

Quetglas, Josep: Le Corbusier y Pierre Jeanneret. Villa Savoye "Les Heures Claires" 1928-1962. Madrid: Ediorial Rueda S.L, 2004 
Quetglas, Josep: “Les Heures Claires. Proyecto y arquitectura en la Villa Savoye de Le Corbusier y Pierre Jeanneret”. Sant Cugat del Valdès: Associaciò d'idees. Centre d'Investigacions Estètiques, 2008

Roth, Alfred: "Bauherr war eine Baudame”. En Aktuelles Bauen Plan N9, 1987.

Sáenz de Oiza, Francisco Javier: Tres arquitecturas. Entrevista TVE. Noviembre, 1990

Savoye, Jean-Marc; DELHOMME, Jean-Philippe: Les Heures Claires de la Villa Savoye. Paris: Éditions des Quatre Chemins, 2015

Siza, Álvaro: Textos. Madrid: Abada ediciones, 2014

Zevi, Bruno: Historia de la Arquitectura Moderna. $5^{\text {a }}$ Ed. Barcelona: Editorial Poseidón, 1980.

Zubiri, Xavier: ¿Qué es investigar?. Discurso en la entrega del Premio Ramón y Cajal. Octubre 1982 